\title{
Editorial
}

\section{The importance of generating evidence on typhoid fever for implementing vaccination strategies}

\author{
Duncan Steele
}

Initiative for Vaccine Research, World Health Organization, Geneva, Switzerland

Vaccines and Immunization, PATH, 1455 Leary Way NW, Seattle, WA 98107, USA

J Infect Developing Countries 2008; 2(4): 250-252.

Received 6 August 2008 - Accepted 6 August 2008

Copyright $\odot 2008$ Steele. This is an open access article distributed under the Creative Commons Attribution License, which permits unrestricted use, distribution, and reproduction in any medium, provided the original work is properly cited.

Typhoid fever, caused by Salmonella enterica serovar Typhi (S. Typhi), remains a major cause of enteric disease and a significant public health problem, predominantly in children in developing countries. Global estimates of typhoid fever range from 17 to 22 million cases per year with an associated 216,000 to 600,000 deaths annually $[1,2]$. This lower estimate is based on a conservative case fatality rate of $1 \%$, (CRF range varies from $1 \%$ to $4 \%$; yet the number of typhoidrelated deaths each year is comparable to that of other diseases that are considered top priorities for disease control in the global health community, including cervical cancer caused by HPV, and is greater than others such as Japanese encephalitis and meningococcal meningitis.

The continued high burden of typhoid fever and the alarming spread of antibiotic resistant strains led the World Health Organization (WHO), almost ten years ago, to recommend immunization using the two new-generation vaccines in schoolaged children in areas where typhoid fever posed a significant problem and where antibiotic resistant strains were prevalent [3]. Nevertheless, with the exception of certain provinces in China, parts of Vietnam and one state in India, this recommendation has yet to be implemented in typhoid-endemic countries.

Several reasons have been given for this lack of uptake of typhoid vaccination in endemic countries, including those gleaned from a survey of policymakers in Asia conducted in 2000-2001 [4]. Most critically, many developing countries were uncertain of their true typhoid disease burden, due to the lack of inexpensive rapid diagnostic tools, infrequency of laboratory testing, poor disease reporting systems, and the fact that the clinical diagnosis of the disease is often confused with other febrile illnesses. In some countries, there has also been political pressure for local government officials not to report typhoid cases, since they are considered an indicator of inadequate or failing water and sanitation systems. There has also been a sense of complacency created by the introduction of relatively inexpensive antibiotics several decades ago, which were effective in substantially reducing typhoid-related deaths, but which have progressively become ineffective due to the acquisition of antibiotic resistance by the bacterium. In addition, with some exceptions, typhoid tends not to cause major epidemics that command the public's attention, but extracts its toll of morbidity and mortality as an endemic illness.

Other barriers to greater public sector use of these vaccines include: the relatively high prices of the vaccines on the world market, although this situation is now changing; a preference among policymakers for water and sanitation improvements over vaccination to control many enteric diseases; a lack of awareness among many policymakers about the new-generation vaccines; and uncertainty of the logistic feasibility of mass vaccination of children outside the infant EPI schedule in their countries. Furthermore, the peak age group for typhoid in many settings is from 5 to 15 years, which falls outside of the under-five year age group that has been the focus 
of most international public health efforts on vaccines.

New data from Asia on the disease and economic burden of typhoid fever, recent trends in antibiotic resistance, and vaccine effectiveness and cost-effectiveness, along with the increased availability of typhoid vaccines and sharp reductions in their price, led WHO to review, update and reinforce its recommendations for the use of typhoid vaccines for populations at high risk, so that this disease can truly be controlled in countries where it remains a significant public health problem [5].

Field studies have provided new data on the magnitude of typhoid disease, especially in Asia, including incidence rates, age distribution patterns and trends in antibiotic resistance of $S$. Typhi, as well as on the economic burden of the disease. Children in poor urban areas in Asia remain at high risk of getting typhoid fever. Prospective disease burden studies in five Asian study sites found annual incidence rates of blood culture-confirmed typhoid fever of 180-494/100,000 among 5-15 year olds in three urban slum areas (North Jakarta, Indonesia; Kolkata, India; and Karachi, Pakistan) [6]. Rates of more than $100 / 100,000$ are considered very high. While these studies confirm that school-aged children are at particularly high risk, pre-school children as young as two years old were shown to also be highly vulnerable with incidence rates that were as high as these.

Studies on the incidence and burden of disease of typhoid in Africa are relatively sparse, in large part because of the limited availability of clinical bacteriology laboratories to perform blood cultures, and lack of inexpensive reliable rapid laboratory diagnostic tools. Nevertheless, several lines of evidence indicate that the disease is prevalent across much of the continent. Data exist from a few population-based surveys and systematic hospital-based studies carried out in Africa, including sub-Sahara Africa. Typhoid has been described as a major health problem in school children in Egypt, Rwanda, Kenya and South Africa. In addition, multidrug resistant strains of $S$. Typhi have emerged in Africa and are increasing in incidence with 75-82\% multidrug resistant strains reported in Kenya. This figure is similar to reports from South Africa, although higher than that reported from Ghana.
Nevertheless, MDR strains have been reported from East, West and southern Africa.

The three studies examining typhoid fever and invasive salmonellosis in Ethiopia, Malawi and Kenya in this edition are extremely important in bridging this gap in knowledge of typhoid fever in the African continent. The burden of disease of typhoid and non-typhoidal Salmonella is not well understood in the region and further efforts are required to elucidate the epidemiology, burden of disease, and strain variation in Africa of these pathogens.

Finally, large vaccine demonstration studies in typhoid-endemic countries of Asia have yielded data on the effectiveness of $\mathrm{Vi}$ vaccine; the feasibility, acceptability and costs of large-scale community- or school-based vaccination; and the population demand for new-generation typhoid vaccines. Demonstration projects using Vi vaccine and Ty21a have shown large-scale communityand school-based vaccination to be feasible and well accepted by populations in endemic countries. Evidence from China suggests that the programmatic use of Vi vaccine in selected areas largely controlled the disease within a four- to fiveyear period, reducing incidence to very low levels. Experience in China has also shown that $\mathrm{Vi}$ vaccination is equally effective in controlling currently occurring typhoid outbreaks as it is in reducing endemic disease. Typhoid incidence varies considerably within countries, underscoring the fact that typhoid control, including vaccination, can focus on high-risk populations instead of being universally applied within countries.

The WHO recommendations for the use of typhoid fever vaccines are described in the recent "Typhoid Vaccines Position Paper" [5]. These include recommendations that in view of the continued high incidence of typhoid fever and increasing antibiotic resistance, and given the safety, efficacy, feasibility and affordability of two licensed vaccines ( $\mathrm{Vi}$ and Ty21a), affected countries should consider programmatic use of typhoid vaccines for controlling endemic disease. In addition, all typhoid vaccination programmes should be implemented in the context of other efforts to control the disease, including health education, water quality and sanitation improvements, and training of health professionals in diagnosis and treatment. Finally, in many countries, the control of the disease will require 
only vaccination targeted to high-risk groups and populations, as opposed to universal vaccination of the population.

Given the importance of information on disease incidence for informing and alerting policy and decision makers, and for targeting vaccination campaigns and assessing impact, priority should be given to strengthening surveillance systems for typhoid fever and to generating robust data on the disease.

\section{References}

1. Ivanoff B, Levine MM, Lambert PH (1994) Vaccination against typhoid fever: present status. Bull WHO 72(6):957-791.

2. Crump JA, Luby SP, Minta ED (2004) The global burden of typhoid fever. Bull WHO 82(5):346-53.

3. World Health Organization. (1999) Strategies, policies and practices for immunization of adolescents: A review. Bull WHO 72(6):957-971

4. DeRoeck D, Clemens JD, Nyamete A, Mahoney RT (2005) Policymakers' views regarding the introduction of new-generation vaccines against typhoid fever, shigellosis and cholera in Asia. Vaccine 23:2762-2774.

5. World Health Organization. (2008) Typhoid Vaccines Position Paper. Weekly Epidemiological Record 83(6):4960.

6. Fondation Merieuex (April 2007) Report of the meeting on "Typhoid Fever, a neglected disease: Towards a vaccine introduction policy". Fondation Merieux, Annecy, France.
Corresponding Author: Duncan Steele, Vaccines and Immunization, PATH, 1455 Leary Way NW, Seattle, WA 98107, USA, Tel: 1-206 788 2458, Fax: 1-206 2856619

Email: dsteele@path.org (Previous address: Initiative for Vaccine Research, World Health Organization, Geneva, Switzerland).

Conflict of interest: No conflict of interest is declared. 